\title{
Using Sliding Mode Control Theory for Radio Resource Management in Wireless Systems
}

\author{
Mihaela R. Cistelecan \\ Department of Automation \\ Technical University of Cluj-Napoca, Romania \\ Email: mihaela.cistelecan@aut.utcluj.ro
}

\author{
Dimitrie C. Popescu \\ Department of Electrical Engineering \\ University of Texas at San Antonio, USA \\ Email: dimitrie.popescu@utsa.edu
}

\begin{abstract}
In this paper we present a theoretical approach to power control for ad-hoc wireless networks using sliding mode control theory. We allow user mobility, as well as addition of new links to the wireless network or removal of inactive links from the network. We derive a dynamic system based on the signal-to-interference ratio (SIR) of links in the network whose control input is related to the transmitted power, and show how sliding mode control theory is used to derive the power equations that lead to an equilibrium point where the link SIRs are optimized.
\end{abstract}

\section{INTRODUCTION}

The increasing demand for wireless services requires a more efficient use of the limited resources that are available in a radio communication system. An important component of radio resource management is transmitter power control, which contributes to minimizing interference, increasing the wireless network capacity, and extending the battery life of active nodes in the wireless network by ensuring that these transmit at the minimum power level necessary to achieve a specified quality of service defined in terms of the SIR.

A good power control mechanism should balance several conflicting requirements which can be summarized as follows:

- The SIR of any node in the wireless network can be increased by increasing its transmitted power.

- Increasing the transmitted power for one node will increase the interference experienced by all the other nodes in the network, which may increase their transmitted powers as well to overcome the increased interference.

- Increasing the transmitted power consumes more energy and shortens the battery life of nodes in the wireless network.

When all nodes keep increasing their transmitted power to meet specified SIR values, they will end up transmitting at their maximum allowed power level which will exhaust their battery energy rapidly. This can create instability in the wireless network which may experience rapid deactivation of nodes due to battery failure.

Two key issues under the scrutiny of the on-going research in power control for wireless networks are users mobility [1], and addition/removal of users to/from the network [2], which should be studied in the context of the conflicting requirements mentioned previously. Traditionally, these two issues have been treated separately, and in an empirical manner. In our paper we propose a systematic approach of these two issues simultaneously, using the mathematical framework of sliding mode control theory [3]. We note that alternative approaches based on control theory were used for analyzing power control algorithms in [4]-[6].

The purpose of the paper is two-fold: 1 . to find a mathematical model for wireless networks that incorporates user mobility as well as network dynamics (addition/removal of user to/from the network); 2 . to design a decentralized sliding mode control law that stabilize the system and meet the quality of service requirements. We present the derivation of the dynamic equation of the SIR error function, and apply sliding mode control theory to obtain a sliding mode control law that will bring the error to the origin through appropriate power updates. The power updates will be designed to allow a maximum number of $N$ active links with specified minimum SIRs in the wireless ad-hoc network. We will also investigate how the sliding mode control can be used in the context of a dynamic network when new links become active and are added to the network, or links that become inactive are removed from the network.

The paper is organized as follows. In the next section we present the main expressions used in power control for wireless networks. In Section III we give the sliding mode control design when the users are assumed to be mobile. In section IV we show some numerical simulations results. In Section V we briefly show how to deal with the admission/removal of users, when the sliding-mode control is used. Conclusions show some of the tracks we still have to investigate. All proofs are gathered into the Appendix that completes the paper.

\section{The Wireless Network Model}

According to [2] an ad-hoc wireless network can be regarded as a collection of interfering wireless links between mobile nodes of the network which apply power control algorithms to improve the quality of the link. As measure of the link quality the SIR at the receiver is used which is expressed as [2], [7]

$$
\gamma_{i}=\frac{G_{i i} p_{i}}{\sum_{j \neq i} G_{i j} p_{j}+\eta_{i}} \quad i, j=1, \ldots, N
$$

where $p_{i}$ is the transmitted power of user $i, G_{i i}$ is the gain of the channel between user $i$ and its corresponding 
receiver, $G_{i j}$ is the gain of the channel between other user $j$ in the system and receiver $i$, and $\eta_{i}$ is the power of the additive white Gaussian noise (AWGN) that corrupts the received signal at receiver $i$. Channel gains are assumed timevarying with known lower and upper bounds. The measurable variables, available to the i-th node are: $\gamma_{i}, G_{i i}, p_{i}, \eta_{i}$.

In this context, for each link $i$, a lower SIR value $\gamma_{i}^{m}$ is imposed, and in order for all links to operate properly it is required that $\gamma_{i} \geq \gamma_{i}^{m}, \forall i$. When the actual SIR of link $i$ is below the specified lower bound $\gamma_{i}^{m}$ the link is not functional as a communication link, but works as a disturbance for all functional links in the network, [2]. When the actual SIR of link $i$ is above $\gamma_{i}^{m}$ but below a pareto-optimal value $\gamma_{i}^{M}$ we need to employ power control to increase it.

By denoting the interference experienced by the link with:

$$
d_{i}=\sum_{j \neq i} G_{i j} p_{j}
$$

and under the assumption that the AWGN is stationary (that is $\eta_{i}=$ constant $\Longrightarrow \dot{\eta}_{i}=0$ ) we obtain by taking the derivative of equation (1):

$$
\dot{\gamma}_{i}=-\frac{\dot{d}_{i}}{\eta_{i}+d_{i}} \gamma_{i}+\frac{\dot{G}_{i i}}{\eta_{i}+d_{i}} p_{i}+\frac{G_{i i}}{\eta_{i}+d_{i}} u_{i}
$$

The pareto-optimal vector of the network $\left(\begin{array}{lll}\gamma_{1}^{M} & \cdots & \gamma_{N}^{M}\end{array}\right)^{T}$ describes the equilibrium of the network for a given configuration $\left[\left(G_{i j}, \dot{G}_{i j}\right)\right]_{i j}$ and should be estimated by design. We assume that $\mathrm{N}$ is the total number of users that the network, once designed, could accommodate. We assume that the transmission power is bounded as $0 \leq p_{i}^{m} \leq p_{i} \leq p_{i}^{M}$ and the SIR is bounded as $0 \leq \gamma_{i}^{m} \leq \gamma_{i} \leq \gamma_{i}^{M}$.

\section{Modeling Power Control Assuming Users MOBILITY}

The proposed update for power will be based on minimization of the error function

$$
e_{i}=\gamma_{i}^{M}-\gamma_{i} ; \quad \gamma_{i} \geq \gamma_{i}^{m}
$$

for all links in the network. Thus, we obtain the following set of equations for link $i=1, \ldots, N$

$$
\left\{\begin{array}{c}
\dot{p}_{i}=u_{i} \\
\dot{e}_{i}=-\frac{\dot{d}_{i}}{\eta_{i}+d_{i}} e_{i}-\frac{\dot{G}_{i i}}{\eta_{i}+d_{i}} p_{i}-\frac{G_{i i}}{\eta_{i}+d_{i}} u_{i}+\frac{\dot{d}_{i}}{\eta_{i}+d_{i}} \gamma_{i}^{M}
\end{array}\right.
$$

which should be controlled to reach an equilibrium point as close to the origin as possible. From equation (1) we note that the quantity $\eta_{i}+d_{i}$ is known for each link based on knowledge of current link SIR $\gamma_{i}$, gain $G_{i i}$, and transmitted power $p_{i}$ :

$$
\frac{1}{\eta_{i}+d_{i}}=\frac{\gamma_{i}}{G_{i i} p_{i}}
$$

Thus, from (5) we note that the user dynamics is parameter time-varying with an exogenous disturbance $\dot{d}_{i}$. Variables $d_{i}$ and $\dot{d}_{i}$ revel the interaction between user $i$ and the other users of the network. The $N$ dynamic systems in (5) are interconnected through variables $d_{i}$ given by

$$
d=\left(\Upsilon-\Upsilon_{\Delta}\right) p
$$

where $\Upsilon=\left[G_{i j}\right]_{i, j}, \Upsilon_{\Delta}=\operatorname{diag}\left(\left[G_{i i}\right]_{i}\right), d^{T}=$ $\left[\begin{array}{lll}d_{1} & \ldots & d_{n}\end{array}\right]$. Taking the derivative of (7) and using that $\dot{p}_{i}=u_{i}$ we get the dynamics of variables $d_{i}$ from:

$$
\dot{d}=\left(\Upsilon-\Upsilon_{\Delta}\right) u+\left(\dot{\Upsilon}-\dot{\Upsilon}_{\Delta}\right) p
$$

Note that, for the control laws to keep the users errors to zero, from (5) with $e_{i}=\dot{e}_{i}=0$, we get

$$
u_{i}^{0}=-\frac{\dot{G}_{i i} p_{i}}{G_{i i}}+\frac{\dot{d}_{i} \gamma_{i}^{M}}{G_{i i}} \quad \forall i
$$

A solution for a sound design of wireless power control, while dealing with the user mobility $\left(\dot{G}_{i j} \neq 0\right)$, is the sliding mode control concept. In order to make this idea possible we append to the system (5) the auxiliary variable $x_{i}$, so that the dynamics of the i-th node is as follows:

$$
\left\{\begin{array}{c}
\dot{p}_{i}=u_{i} \\
\dot{e}_{i}=f_{i}^{e}-\frac{\gamma_{i}}{p_{i}} u_{i} \quad ; \quad f_{i}^{e}=\frac{\gamma_{i}}{G_{i i} p_{i}}\left(\dot{d}_{i} \gamma_{i}-\dot{G}_{i i} p_{i}\right) \\
\dot{x}_{i}=f_{i}(\cdot)
\end{array}\right.
$$

We use the following sliding manifolds:

$$
s_{i}=e_{i}+c_{i} x_{i}=0 \quad \forall i
$$

and look for both the proper appended dynamics $\dot{x}_{i}=f_{i}(\cdot)$ and proper decentralized controls, $u_{i}(t)$, able to stabilize the wireless network dynamics into an equilibrium.

Proposition 1: The wireless network consisting of $\mathrm{N}$ dynamical nodes (10), with the connectivity matrix $A=$ $\left[A_{i j}\right]_{i, j=1, . . N}$, as follows:

$$
\begin{gathered}
A_{i i}=\frac{\gamma_{i}}{G_{i i} p_{i} s_{i}}\left(G_{i i} u_{i}+\dot{G}_{i i} p_{i}-c_{i} \frac{G_{i i} p_{i}}{\gamma_{i}} \dot{x}_{i}\right) ; \\
A_{i j}=-\frac{\gamma_{i}^{2}}{G_{i i} p_{i}} \frac{1}{s_{j}}\left(G_{i j} u_{j}+\dot{G}_{i j} p_{j}\right)
\end{gathered}
$$

may be stabilized by a sliding mode control law, defined on (11), if the following requirements are fulfilled:

(P1.1). The diagonal entries in the connectivity matrix (12) are positive $-A_{i i}>0$ - that is:

$$
\frac{1}{s_{i}}\left(G_{i i} u_{i}+\dot{G}_{i i} p_{i}-c_{i} \frac{G_{i i} p_{i}}{\gamma_{i}} \dot{x}_{i}\right)>0
$$

(P1.2). The diagonally dominance condition for the connectivity matrix (12) - $\left|A_{i i}\right|>\sum_{j}\left|A_{i j}\right|$ - is fulfilled, that is:

$$
\left|\frac{1}{\gamma_{i} s_{i}}\left(G_{i i} u_{i}+\dot{G}_{i i} p_{i}-c_{i} \frac{G_{i i} p_{i}}{\gamma_{i}} \dot{x}_{i}\right)\right|>\sum_{j}\left|\frac{G_{i j} u_{j}+\dot{G}_{i j} p_{j}}{s_{j}}\right|
$$

(P1.3). The appended dynamics, $f_{i}(\cdot)$, is consistent with the sliding mode reaching condition with disturbance rejection;

(P1.4). The appended dynamics is properly constrained;

(P1.5). The dynamics of the $\mathrm{i}$-th node is constrained on the sliding manifold (11), as follows:

$$
s_{i}=0 \rightarrow\left|c_{i} f_{i}(\cdot)\right|<\gamma_{i}^{M}
$$

(P1.6). The appended dynamics may guarantee $x_{i}=\dot{x}_{i}=$ 0 when $s_{i}=\dot{s}_{i}=0$ and $e_{i}=\dot{e}_{i}=0$. 
(1.7). The power does not saturate, that is:

$$
u_{i}<0 \quad \text { if } \quad p_{i}=p_{i}^{M} \quad \text { and } \quad u_{i}>0 \quad \text { if } \quad p_{i}=p_{i}^{m}
$$

Proof: See Appendix.

Proposition 2: The wireless network consisting of $N$ dynamical nodes (10) and the following appended dynamics:

$$
\begin{gathered}
\dot{x}_{i}=f_{i}^{x}+\frac{\gamma_{i}}{c_{i} p_{i}} u_{i} \\
f_{i}^{x}=-\frac{\gamma_{i}}{G_{i i} p_{i}} s_{i}+\frac{\dot{G}_{i i} \gamma_{i}}{c_{i} G_{i i}}-\frac{\gamma_{i}}{G_{i i} p_{i}} k_{i} \operatorname{sgn}\left(s_{i}\right)
\end{gathered}
$$

may be stabilized, on the sliding manifolds (11), by a decentralized control law, that fulfills the conditions (P1.3)(P1.7), if:

$$
\begin{gathered}
k_{i}>0 \\
c_{i}>\frac{\gamma_{i}^{M}}{\varsigma} \sum_{j} G_{i j}^{\max }\left(p_{j}^{M}-p_{j}^{m}\right)+\left|\dot{G}_{i j}^{\max }\right| p_{j}^{M}>0
\end{gathered}
$$

where $\varsigma \ll 1$.

Proof: see Appendix.

Note that for the appended dynamics chosen as in (17), the dynamics of the sliding variable is as follows:

$$
\dot{s}_{i}=\dot{e}_{i}+c_{i} \dot{x}_{i}=f_{i}^{e}+c_{i} f_{i}^{x}
$$

and, consequently, the control variable $u_{i}$ does not influence the expression of the first derivative of the sliding variable. Still, the discontinuous term $k_{i} \operatorname{sgn}\left(s_{i}\right)$ from the appended dynamics, if properly designed, is able to cope with the unknown disturbance of the i-th node. Therefore, the control variable $u_{i}$ may be used to smoothly adjust the power value.

Proposition 3: The following control law:

$$
u_{i}(t)=-\frac{\dot{G}_{i i}(t)}{G_{i i}(t)} p_{i}(t)+\hat{u}_{i}(t)
$$

satisfies the conditions (P1.3)-(P1.6) for the wireless network of the Proposition 2, as follows:

(P3.1). The conditions (P1.3)-(P1.4) are fulfilled if either:

$$
k_{i}>\max \left\{\frac{\gamma_{i}^{M}}{c_{i}}\left|\dot{d}_{i}^{\max }\right|, \frac{G_{i i}^{\max }}{c_{i}} \frac{\left(p_{i}^{M}\right)^{2}}{p_{i}^{m}} \frac{\gamma_{i}^{M}}{\gamma_{i}^{m}}\right\}
$$

or

$$
\begin{gathered}
k_{i}>\max \left\{\frac{\gamma_{i}^{M}}{c_{i}}\left|\dot{d}_{i}^{\max }\right|, G_{i i}^{\max } p_{i}^{M} \frac{\gamma_{i}^{M}}{c_{i} \gamma_{i}^{m}}\right\} \quad \text { and } \\
\operatorname{sgn}\left(\hat{u}_{i}\right)=-\operatorname{sgn}\left(s_{i}\right)
\end{gathered}
$$

(P3.2). The conditions (P1.5)-(P1.6) are fulfilled if

$$
\begin{gathered}
\hat{u}_{i}(t)=-\varepsilon_{i}(t) x_{i}(t)+a_{i}(t) s_{i}(t)+b_{i}(t) \operatorname{sgn}\left(s_{i}\right) \\
\left|c_{i} \varepsilon_{i}(t) x_{i}(t)\right|<\gamma_{i}^{M}
\end{gathered}
$$

where $\varepsilon_{i}(t), a_{i}(t), b_{i}(t)$ are either constants or functions depending on the i-th node (known) variables, and $\varepsilon_{i}>0$, $\varepsilon_{i} \neq 0$.

Proof: see Appendix.

Proposition 4: For the wireless network given in Proposition 2, the saturation problem (P1.7) is solved by the following control:

$$
\begin{gathered}
\tilde{u}_{i}=\operatorname{sgn}\left(p_{i}-p_{i}^{M}\right) \operatorname{sgn}\left(p_{i}^{m}-p_{i}\right) u_{i}+ \\
+\left(\operatorname{sgn}\left(p_{i}^{M}-p_{i}\right)-1\right)\left|u_{i}\right|+\left(1+\operatorname{sgn}\left(p_{i}^{m}-p_{i}\right)\right)\left|u_{i}\right|
\end{gathered}
$$
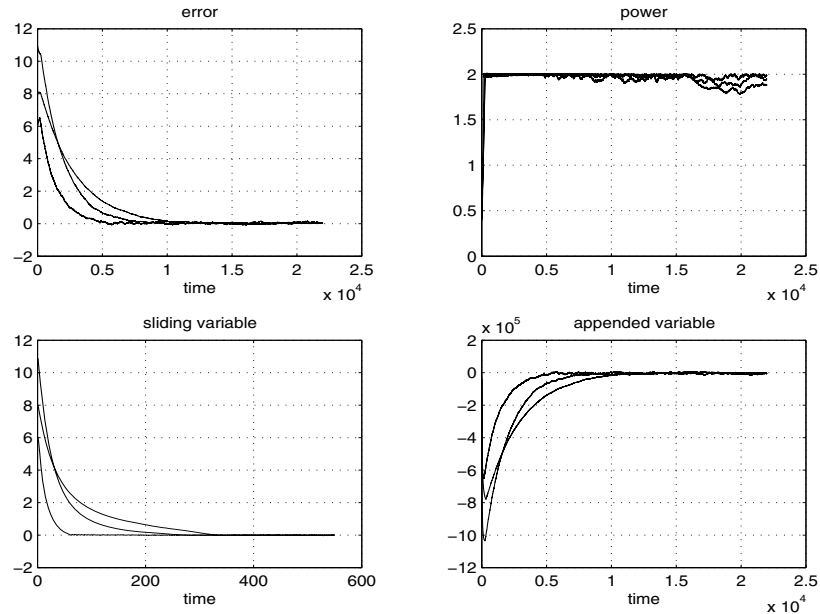

Fig. 1. Simulation results

where $u_{i}$ is as in Proposition 3, with the gain $k_{i}$ as follows:

$$
k_{i}=\tilde{k}_{i}+\frac{\left|\dot{G}_{i i}\right|}{G_{i i}} p_{i}^{M}
$$

where $\tilde{k}_{i}$ is chosen as the gain $k_{i}$ in (22).

Proof: see Appendix.

Theorem 1: The wireless network given by the Propositions 2 and 4 is stabilized to an equilibrium point by a sliding mode control (26), (21), (24) with $a_{i}=b_{i}=0$ and $\varepsilon(t)=$ const. if:

(T1).

$$
c_{i}<\frac{\gamma_{i}^{M}}{p_{i}^{M}\left(1+\frac{\left|\dot{G}_{i i}^{m a x}\right|}{G_{i i}^{m i n}}\right)-p_{i}^{m}}
$$

(T2).

$$
\left|x_{i}(0)\right|<\frac{\left|e_{i}(0)\right|}{c_{i}} ; \quad \operatorname{sign}\left(x_{i}(0)\right)=-\operatorname{sign}\left(e_{i}(0)\right) ;
$$

(T3).

$$
\varepsilon_{i}<\frac{\min \left\{\left|\frac{\gamma_{i}^{M}}{c_{i}}-\right| w_{i}^{M}||,\left|p_{i}^{M}-p_{i}^{m}+\frac{\left|\dot{G}_{i i}^{\min }\right|}{G_{i i}^{m a x}} p_{i}^{m}-\right| w_{i}^{M}||\right\}}{x_{i}(0)}
$$

where $w_{i}^{M}=\frac{\gamma_{i}^{M}}{G_{i i}^{m i n} p_{i}^{m}}\left(k_{i}+e_{i}(0)+c_{i} x_{i}(0)\right)$

Proof: see Appendix.

\section{NUMERICAL SIMULATIONS}

In order to test the validity of the proposed sliding mode control scheme we have performed numerical simulations. The gains $G_{i j}$ were considered time-varying as follows $G_{i j}(t)=\varrho\left(d_{i j}^{4}(t)\right)^{-1}$ where $\varrho=0.097$ and $d_{i}$ is the distance from the user $i$ to the base $j$. The gain modification was based on the "random" function, as follows: $\dot{G}_{i i}=\epsilon G_{i i}$, where $\epsilon \in[-1,1]$ is a random number. We assumed the distance bounds as $10 m \leq d_{i} \leq 10000 m, p_{i}^{m}=0.1 \mathrm{Watts}$, $p_{i}^{M}=2$ Watts, $\gamma_{i}^{m}=1, \gamma_{i}^{M}=12$. The simulations were performed for a "fixed assignment", [7], scenario, with a single base and three users, using the Euler integration algorithm. We used the control law from Theorem 1. From Figure 1 we see that the errors vanish eventually, while the power is constrained to the imposed range of variation. 


\section{ADMISSION/REMOVAL OF USERS TO/FROM THE WIRELESS NETWORK}

In this section we only give an idea about how to deal with a time-varying number of users. Like in [2] we divide the users in two groups, as follows: $\mathcal{A}_{k}$ - the group of users with $\gamma_{i} \geq \gamma_{i}^{m}$ (active users) and $\mathcal{B}_{k}$ - the group of users with $\gamma_{i}<\gamma_{i}^{m}$. The users from $\mathcal{B}_{k}$ do not "feel" the users from $\mathcal{A}_{k}$, but the users from $\mathcal{A}_{k}$ are disturbed by users from $\mathcal{B}_{k}$. The users from $\mathcal{A}_{k}$ should cooperate with users from $\mathcal{B}_{k}$ in order to let them enter the network without disrupting the "team" of users from $\mathcal{A}_{k}$. The power control is given as follows:

$$
\begin{array}{llll}
\dot{p}_{i}=v_{i} & \text { if } & i \in \mathcal{A}_{k} & (\text { closed }- \text { loop control }) \\
\dot{p}_{i}=\delta_{i} & \text { if } & i \in \mathcal{B}_{k} & (\text { open }- \text { loop control })
\end{array}
$$

where $\delta_{i}$ are constants that should be estimated. The index $k$ for the sets $\mathcal{A}_{k}$ and $\mathcal{B}_{k}$ stands for the topology of the network at discrete time $k$. Each user may interact with a different number of other users at different time moments, $\mathrm{k}$. It is worth noted that removal / admission of users into a wireless network is translated to a dynamical network undergoing a switching topology.

Essentially, the removal of a link can be either the result of forced disconnection, of one or many users, in order to maintain the SIR of the other users above $\gamma_{i}^{m}$, or the result of a giving-up to connection (drop out) coming from the user. In the case of a forced disconnection there has to be a well-stated method to choose which users are disconnected.

The complexity of the power control for wireless networks when the number of users is time-varying is challenging. It comes up in two distinguished cases:

1. Users mobility makes the interaction among users and bases to be limited to a finite geographically stated horizon; if the $\mathrm{j}$-th user is far away from the $\mathrm{i}$-th base then $G_{i j}=0$. The proper functioning of the network (the active links are not removed) should be guaranteed in cases when $G_{i j}\left(k_{1}\right) \neq 0$, $G_{i j}\left(k_{2}\right)=0, G_{i j}\left(k_{3}\right) \neq 0$, for $k_{1}<k_{2}<k_{3}$ consecutive time instances.

2. If the network is in an equilibrium point or in a transient towards an equilibrium point: a). the admission of a new link must not remove any other link; b). a drop out of a link should end into an equilibrium point without power saturation.

Taking into account the heuristics given in [2] we construct a virtual dynamics of the wireless network that allows us to mathematically model the ideas of admission / removal of users. Thus, we add two new terms to the dynamics of the i-th SIR, as follows:

$$
\begin{gathered}
\dot{p}_{i}=u_{i}(t) \\
\dot{\gamma}_{i}(t)=-\frac{\dot{d}_{i}(t)}{\eta_{i}+d_{i}(t)} \gamma_{i}(t)+\frac{\dot{G}_{i i}(t)}{\eta_{i}+d_{i}(t)} p_{i}(t)+\frac{G_{i i}(t)}{\eta_{i}+d_{i}(t)} u_{i}(t) \\
+\alpha_{i} \gamma_{i}\left(t-\tau_{1}\right)-\beta_{i} \gamma_{i}\left(t-\tau_{2}\right)
\end{gathered}
$$

with $\tau_{1}<\tau_{2}, \alpha_{i}>0, \beta_{i}>0$.

The reasoning beyond the virtual dynamics (32) is as follows:

" $\alpha_{i} \gamma_{i}\left(t-\tau_{1}\right)$ ": When entering the network the i-th user is accommodated in a time interval $\tau_{1}$, by receiving a little more power. Thus, during the accommodation period of time its SIR is prevented from switching many times between $\gamma_{i}<\gamma_{i}^{m}$ and $\gamma_{i}>\gamma_{i}^{m}$.

" $-\beta_{i} \gamma_{i}\left(t-\tau_{2}\right)$ ": On the other hand, if $\gamma_{i}(t)>\gamma_{i}^{m}$ for all $t>\tau_{2}$, since the $\mathrm{i}$-th user was given a limited horizon $\tau_{1}+\tau_{2}$ for communication, it should cease communication (should be penalized through control) to give the chance of connection to other users, if and only if the network is so busy that joining of new users is possible only by disconnecting some of the old users. Thus, the last term introduced in (32) is intended to hinder a too fast increasing of the SIR $\gamma_{i}$ towards the target $\gamma_{i}^{M}$, giving a chance to other users to be accommodated by the network, as well.

The users from $\mathcal{B}_{k}$, waiting to join the network, are included as disturbances for the network nodes $\mathcal{A}_{k}$, hence in (32):

$$
\begin{array}{ll}
d_{i}=d_{i}^{\mathcal{A}}+d_{i}^{\mathcal{B}} ; & d_{i}^{\mathcal{A}}=\sum_{j \in \mathcal{A}_{k}} G_{i j} p_{j} \\
d_{i}^{\mathcal{B}}=\sum_{j \in \mathcal{B}_{k}} G_{i j} p_{j}
\end{array}
$$

From (32) and (33), the error dynamics for the virtual network is as follows:

$$
\begin{gathered}
\dot{e_{i}}=\frac{\dot{d}_{i}^{\mathcal{A}}+\dot{d}_{i}^{\mathcal{B}}}{\eta_{i}+d_{i}^{\mathcal{A}}+d_{i}^{\mathcal{B}}}\left(\gamma_{i}^{M}-e_{i}\right)-\frac{\dot{G}_{i i}}{\eta_{i}+d_{i}^{\mathcal{A}}+d_{i}^{\mathcal{B}}} p_{i}- \\
\frac{G_{i i}}{\eta_{i}+d_{i}^{\mathcal{A}}+d_{i}^{\mathcal{B}}} u_{i}-\alpha_{i} e_{i}\left(t-\tau_{1}\right)+\beta_{i} e_{i}\left(t-\tau_{2}\right)+\left(\alpha_{i}-\beta_{i}\right) \gamma_{i}^{M}
\end{gathered}
$$

A quick analysis of (34) revels the fact that working on the virtual network dynamics, when a user first connects $\left(\gamma_{i} \simeq \gamma_{i}^{m}\right)$ the term $-\alpha_{i} e_{i}\left(t-\tau_{1}\right)$ helps the error vanish. On the other hand, after $\tau_{1}+\tau_{2}$, the term $\beta_{i} e_{i}\left(t-\tau_{2}\right)$ will make the error rise, hence the SIR is decreased in order to accommodate in the network other users as well. Still, we have no choice to work with the virtual dynamics (32). The choice we are left with is to work on the real dynamics of the wireless network, assuming the i-th node dynamics from the previous section:

$$
\dot{p}_{i}=u_{i} ; \quad \dot{e}_{i}=f_{i}^{e}-\frac{\gamma_{i}}{p_{i}} v_{i} ; \quad \dot{x}_{i}=f_{i}^{x}+\frac{\gamma_{i}}{c_{i} p_{i}} v_{i} ;
$$

with the modified control law:

$$
v_{i}=u_{i}+\frac{p_{i}}{\gamma_{i}}\left(\alpha_{i} \gamma_{i}\left(t-\tau_{1}\right)-\beta_{i} \gamma_{i}\left(t-\tau_{2}\right)-\left(\alpha_{i}-\beta_{i}\right) \gamma_{i}^{M}\right)
$$

where $u_{i}$ is given as in the previous section. The design of the power control for the wireless network should be done for a maximum of $N$ users with specified target SIRs. Then, an investigation on how the network switches from $N_{1}$ active users to $N_{2}$ active users, $N_{1}, N_{2}<N$, should be performed. Similar to the previous section, by applying the sliding mode control, the stability of the switching network - that result by admission/removal of users - depends on the sliding variables associated with each node of the network. Taking into account the disturbance (33), the stability of the network is given by the connectivity matrix $A$, partitioned as follows:

$$
\left[\begin{array}{ll}
A^{\mathcal{A A}} & A^{\mathcal{A B}} \\
A^{\mathcal{B} \mathcal{A}} & A^{\mathcal{B B}}
\end{array}\right]>0
$$

where: $A^{\mathcal{A A}}=\left[A_{i j}\right], i, j \in \mathcal{A}_{k} ; A^{\mathcal{B B}}=\left[A_{i j}\right], i, j \in \mathcal{B}_{k}$; $A^{\mathcal{A B}}=\left[A_{i j}\right], i \in \mathcal{A}_{k}, j \in \mathcal{B}_{k}$, see Appendix. The design 
phase should provide proper values for the open-loop control, $\delta_{i}$, and also, for the following parameters: $\alpha_{i}, \beta_{i}, k_{i}, c_{i}, \varepsilon_{i}$ and $x_{i}(0)$. The design should fulfill the same requirements given in Proposition 1.

\section{CONCLUSIONS}

In order to simultaneously study both the problem of user mobility and addition/removal of users to/ from a wireless network we have applied the sliding mode control theory to the wireless network power control. We derived a continuous time model for the network dynamics and appended a new variable to the real model of the node. The appended variable contributes with an integral effect to both the control and sliding variables. The design is based on sufficient conditions and their necessity is a matter of future investigations. In future work we also plan to investigate further the proposed approach in the context of dynamic networks in which the number of active links in the network varies in time. Moreover, further investigations will be performed for a fixed assignment scheme with change of the assigned base depending on the distance user-base. The proposed control algorithm will be also tested on the "macro-diversity", [7], scenario.

\section{APPENDIX}

\section{A. Proof for Proposition 1}

(P1.1)-(P1.2). Using (5), (6), (8), by imposing the reaching condition for MIMO systems to the wireless network, as follows:

$$
\begin{gathered}
\sum_{i=1}^{N} \varsigma_{i} \mathrm{~s}_{i} \dot{s}_{i}<0 \quad \varsigma_{i}>0 \\
s_{i} \dot{s}_{i}=s_{i} \frac{\gamma_{i}}{G_{i i} p_{i}}\left(\sum_{j \neq i} \gamma_{i} G_{i j} u_{j}-G_{i i} u_{i}+\right. \\
\left.\sum_{j \neq i} \gamma_{i} \dot{G}_{i j} p_{j}-\dot{G}_{i i} p_{i}+c_{i} \frac{G_{i i} p_{i}}{\gamma_{i}} \dot{x}_{i}\right)
\end{gathered}
$$

it turns out that the stability of the network is given by the matrix A given in (12). From [8, p. 349] we have that if all main diagonal entries of a diagonally dominant matrix $\mathrm{A}$ are positive, then all the eigenvalues of $\mathrm{A}$ have positive real part. Taking into account that $G_{i i}>0, p_{i}>0, \gamma_{i}>0$ the positiveness of the diagonal entries $A_{i i}>0$ requires (13). The condition of diagonally dominance on the matrix $\mathrm{A}$ is given by the inequalities (14).

(P1.3). From (38) it turns out that the appended dynamics of the i-th node should fulfill the sliding mode reaching condition, that is $s_{i} \dot{s}_{i}=s_{i}\left(\dot{e}_{i}+c_{i} f_{i}(\cdot)\right)<0$. Since, from (10), $\dot{e}_{i}$ depends on the unknown disturbance $\dot{d}_{i}$, and the control variable $u_{i}$ is constrained by the saturation problem, the function $f_{i}(\cdot)$ should be able to reject the disturbance.

(P1.4). From (10)-(11) we note that the disturbance variation may be expressed as follows:

$$
\begin{gathered}
\dot{d}_{i}=\frac{p_{i} G_{i i}}{\gamma_{i}^{2}} \dot{e}_{i}+\frac{\dot{G}_{i i} p_{i}+G_{i i} u_{i}}{\gamma_{i}}=\frac{p_{i} G_{i i}}{\gamma_{i}^{2}} \dot{s}_{i}-\frac{p_{i} G_{i i}}{\gamma_{i}^{2}} c_{i} f_{i}(\cdot)+ \\
+\frac{\dot{G}_{i i} p_{i}+G_{i i} u_{i}}{\gamma_{i}}
\end{gathered}
$$

If we assume $-\gamma_{i}^{M}<\dot{e}_{i}<\gamma_{i}^{M}$, then, from (40), the appended dynamics should be so that:

$$
-\gamma_{i}^{M}<\dot{s}_{i}-c_{i} f_{i}(\cdot)<\gamma_{i}^{M}
$$

(P1.5). When $s_{i}=\dot{s}_{i}=0$, from (41) the constraint (15) is required.

(P1.6). When $s_{i}=\dot{s}_{i}=0$ and also $\dot{d}_{i}=0$, the i-th node dynamics should be able to reach the equilibrium $\left(e_{i}=\right.$ $0, x_{i}=0$ ), hence $x_{i}=0$ should be an attractor for the appended dynamics $\dot{x}_{i}-f_{i}(\cdot)=0$.

(P1.7). In order to study the power saturation problem we discretize the dynamics: $\dot{p}_{i}=u_{i}$, as in $p_{i}(k+1)=p_{i}(k)+$ $\tau u_{i}, \tau>0$. Thus, it is obvious that we should constrain $u_{i}<$ 0 whenever $p_{i}(k)=p_{i}^{M}$ and $u_{i}>0$ whenever $p_{i}(k)=p_{i}^{m}$.

\section{B. Proof for Proposition 2}

By replacing (17)-(19) into (13), it turns out that $\inf _{s_{i}} \frac{1}{s_{i}}\left(G_{i i} u_{i}+\dot{G}_{i i} p_{i}-c_{i} \frac{G_{i i} p_{i}}{\gamma_{i}} \dot{x}_{i}\right)=\inf _{s_{i}}\left(c_{i}+\frac{c_{i} k_{i}}{\left|s_{i}\right|}\right)=c_{i}>0$

Therefore $A_{i i}>0 \forall i$ and the condition (P1.1) is fulfilled. Moreover (14) becomes as follows:

$$
\inf _{s_{i}, \gamma_{i}}\left|\frac{1}{\gamma_{i} s_{i}}\left(G_{i i} u_{i}+\dot{G}_{i i} p_{i}-c_{i} \frac{G_{i i} p_{i}}{\gamma_{i}} \dot{x}_{i}\right)\right|=\frac{c_{i}}{\gamma_{i}^{M}}
$$

By choosing the constants $c_{i}$ properly, high enough, the condition (P1.2) may be fulfilled if

$$
\frac{c_{i}}{\gamma_{i}^{M}}>\sum_{j}\left|\frac{G_{i j} u_{j}+\dot{G}_{i j} p_{j}}{s_{j}}\right|
$$

Using (14),(42) and the fact that $\left|u_{j}\right|<p_{j}^{M}-p_{j}^{m}$ we compute the supremum for the value $c_{i}$ in (14) taking into account that:

$$
\left|\frac{G_{i j} u_{j}+\dot{G}_{i j} p_{j}}{s_{j}}\right|<\left|\frac{G_{i j}^{\max }\left(p_{j}^{M}-p_{j}^{m}\right)+\left|\dot{G}_{i j}^{\max }\right| p_{j}^{M}}{s_{j}}\right|=\frac{\eta_{i j}}{\left|s_{j}\right|}
$$

as follows:

$$
c_{i}>\frac{\gamma_{i}^{M}}{\varsigma} \sum_{j} \eta_{i j}
$$

where $\varsigma \ll 1$ stands for $\left|s_{j}\right| \approx 0$.

\section{Proof for Proposition 3}

(P3.1). From (10), (17)-(19), (20) the sliding variable dynamics is as follows:

$$
\dot{s}_{i}=\frac{\gamma_{i}^{2}}{G_{i i} p_{i}} \dot{d}_{i}-\frac{c_{i} \gamma_{i}}{G_{i i} p_{i}} s_{i}-\frac{c_{i} \gamma_{i}}{G_{i i} p_{i}} k_{i} \operatorname{sgn}\left(s_{i}\right)
$$

If we impose: $s_{i} \dot{s}_{i}<-\frac{c_{i} \gamma_{i}}{G_{i i} p_{i}} s_{i}^{2}<0$, the gain $k_{i}$ is required to be:

$$
k_{i}>\frac{\gamma_{i}^{M}}{c_{i}}\left|\dot{d}_{i}^{\max }\right|
$$

Therefore, condition (P1.3) is fulfilled by the appended dynamics (17)-(19) if the gain $k_{i}$ is chosen as in (47). In order to guarantee the fulfillment of the condition (P1.4), (17)-(18) and (21) are replaced into (41). Thus, we get the following condition that $\hat{u}_{i}$ should fulfill:

$$
-\gamma_{i}^{M}<\dot{s}_{i}+\frac{c_{i} \gamma_{i}}{G_{i i} p_{i}} s_{i}+\frac{c_{i} \gamma_{i}}{G_{i i} p_{i}} k_{i} \operatorname{sgn}\left(s_{i}\right)-\frac{\gamma_{i}}{p_{i}} \hat{u}_{i}<\gamma_{i}^{M}
$$


Since $\dot{p}_{i}=u_{i}, \hat{u}_{i}$ should be constraint by $\left|\hat{u}_{i}\right|<p_{i}^{M}-p_{i}^{m}$. Therefore, in order for the reaching condition $s_{i} \dot{s}_{i}<0$ to be fulfilled again, the gain $k_{i}$ should be chosen either as in (22) or (23).

(P3.2). By replacing (17)-(19) into (40) we get the following expression for the disturbance:

$$
\dot{d}_{i}=\frac{p_{i} G_{i i}}{\gamma_{i}^{2}} \dot{s}_{i}+\frac{c_{i}}{\gamma_{i}} s_{i}+\frac{c_{i} k_{i}}{\gamma_{i}} \operatorname{sgn}\left(s_{i}\right)
$$

From (49), it is obvious, that $\dot{d}_{i}=0$ should imply both $s_{i}=0$ and $\dot{s}_{i}=0$. The $\left(e_{i}, x_{i}\right)$ dynamics is as follows:

$$
\begin{gathered}
\dot{e}_{i}=\frac{\gamma_{i}^{2}}{G_{i i} p_{i}} \dot{d}_{i}-\frac{\gamma_{i}}{p_{i}} \hat{u}_{i} \\
\dot{x}_{i}=\frac{\gamma_{i}}{G_{i i} p_{i}} s_{i}-\frac{\gamma_{i}}{G_{i i} p_{i}} k_{i} \operatorname{sgn}\left(s_{i}\right)+\frac{\gamma_{i}}{c_{i} p_{i}} \hat{u}_{i}
\end{gathered}
$$

If $\dot{d}_{i}=0$ and $s_{i}=0$, since the dynamics $\dot{x}_{i}=-\varepsilon_{i} x_{i}$, $\varepsilon_{i}>0$, is stable, the equilibrium $\left(e_{i}, x_{i}\right)=(0,0)$ may be reached.

\section{Proof for Proposition 4}

From (21), it is obvious that if we impose $\left|\hat{u}_{i}\right|>$ $p_{\text {max }}\left|\dot{G}_{i i}\right| / G_{i i}$, the condition (P1.6) is re-written as follows:

$$
\hat{u}_{i}<0 \quad \text { if } \quad p_{i}=p_{i}^{M} \quad \text { and } \quad \hat{u}_{i}>0 \quad \text { if } \quad p_{i}=p_{i}^{m}
$$

Note that from (17)-(18), (21), (24), the dynamics of the appended variable $x_{i}$ is as follows:

$$
\dot{x}_{i}+\varepsilon_{i}(t) x_{i}+w_{i}(t)=0 ;
$$

where $w_{i}(t)=w_{i}^{1} s_{i}+w_{i}^{2} \operatorname{sgn}\left(s_{i}\right)$. Since in the steady state $x_{i}(t)=-w_{i}(t) / \varepsilon_{i}(t)$ it is obvious that the sign of the variable $x_{i}$ will follow the sign of the variable $-s_{i}$. Therefore, the choice of the control (21),(23) would be convenient if the bounds $\left(p_{i}=p_{i}^{m}\right.$ or $\left.p_{i}=p_{i}^{M}\right)$ are not reached during the transient. Since from (52) the dynamics of the appended variable may be approximated by

$$
x_{i}(t)=x_{i}(0) e^{-\int_{0}^{t} \varepsilon_{i}(s) d s}-\int_{0}^{t} w_{i}(r) e^{-\int_{r}^{t} \varepsilon_{i}(s) d s} d r
$$

the transient could be slow because it depends on the values of $\varepsilon_{i}(t)$ and $w_{i}(t)$. Moreover, (48) does not guarantee that the case $p_{i}=p_{i}^{M}, \hat{u}_{i}>0, s_{i}<0, \dot{s}_{i}>0$ or the other way around would never be reached. Since there is no such guarantee, when the power saturation is critical, we should consider the case with (21), (22) and choose the control $u_{i}$ as in (26). This is a conservative case and, in order to guarantee (48), a new gain $k_{i}$, as in (27), is required.

\section{E. Proof for Theorem 1}

(T1). From (15), when $s_{i}=0$, the constraint $\left|c_{i} \dot{x}_{i}\right|=$ $\left|c_{i} \varepsilon_{i} x_{i}\right|<\gamma_{i}^{M}$ should be fulfilled. On the other hand as

$$
\left|\dot{p}_{i}\right|=\left|-\frac{\dot{G}_{i i}}{G_{i i}} p_{i}-\varepsilon_{i} x_{i}\right|<p_{i}^{M}-p_{i}^{m}
$$

it turns out that $c_{i}\left|\varepsilon_{i} x_{i}\right|<c_{i}\left(p_{i}^{M}-p_{i}^{m}+\frac{\left|\dot{G}_{i i}^{\max }\right|}{G_{i i}^{m i n}} p_{i}^{M}\right)<\gamma_{i}^{M}$ and, consequently (28) should be fulfilled.

(T2) Since $x_{i}$ and $s_{i}$ have opposite signs, (see (52)), $s_{i}$ should have the sign of $e_{i}$. Therefore, $x_{i}(0)$ should be chosen as in (29).
(T3) Using $w_{i}^{M}=\sup _{t} w(t)$, (see (52) and (18)), we estimate the following function:

$$
x_{i}(t)=x_{i}(0) \exp \left(-\varepsilon_{i} t\right)+\frac{w_{i}^{M}}{\varepsilon_{i}}\left(\exp \left(-\varepsilon_{i} t\right)-1\right)
$$

From (15) and (54) we find a bound for $x_{i}(0)$ as follows:

$$
\left|\varepsilon_{i} x_{i}(0)\right|+\left|w_{i}^{M}\right|<\frac{\gamma_{i}^{M}}{c_{i}}
$$

From the limitation on the variation of power:

$$
\left|\varepsilon_{i} x_{i}(0)\right|+\left|w_{i}^{M}\right|<p_{i}^{M}-p_{i}^{m}+\frac{\left|\dot{G}_{i i}\right|}{G_{i i}} p_{i}
$$

From (55) and (56) the variable $\varepsilon_{i}$ can be chosen as in (30).

F. Partitions of the connectivity matrix for admission/removal of nodes

Using the fact that for all users $j \in \mathcal{B}_{k} s_{j}=e_{j}=\gamma_{i}^{m}-$ $\gamma_{i}>0$, we have:

$$
\begin{gathered}
A_{i i}=\frac{\gamma_{i}}{G_{i i} p_{i} s_{i}}\left(G_{i i} v_{i}+\dot{G}_{i i} p_{i}-c_{i} \frac{G_{i i} p_{i}}{\gamma_{i}} \dot{x}_{i}\right) \in A^{\mathcal{A A}} ; \\
A_{i j}=-\frac{\gamma_{i}^{2}}{G_{i i} p_{i}} \frac{1}{s_{j}}\left(G_{i j} v_{j}+\dot{G}_{i j} p_{j}\right) \in A^{\mathcal{A} \mathcal{A}} \\
A_{i j}=-\frac{\gamma_{i}^{2}}{G_{i i} p_{i}}\left(G_{i j} \delta_{j}+\dot{G}_{i j} p_{j}\right) \frac{1}{\gamma_{j}^{m}-\gamma_{j}} \in A^{\mathcal{A B}}
\end{gathered}
$$

and

$$
\begin{gathered}
A_{i i}=\frac{\gamma_{i}}{G_{i i} p_{i}}\left(G_{i i} \delta_{i}+\dot{G}_{i i} p_{i}\right) \frac{1}{\gamma_{i}^{m}-\gamma_{i}} \in A^{\mathcal{B B}} \\
A_{i j}=-\frac{\gamma_{i}^{2}}{G_{i i} p_{i}} \frac{1}{s_{j}}\left(G_{i j} v_{j}+\dot{G}_{i j} p_{j}\right) \in A^{\mathcal{B A}} \\
A_{i j}=-\frac{\gamma_{i}^{2}}{G_{i i} p_{i}}\left(G_{i j} \delta_{j}+\dot{G}_{i j} p_{j}\right) \frac{1}{\gamma_{j}^{m}-\gamma_{j}} \in A^{\mathcal{B B}}
\end{gathered}
$$

In order to have $A_{i i}>0$ for $i \in \mathcal{B}_{k}$ in (58), the increment $\delta_{i}$ should be chosen either as a constant $\delta_{i}>\frac{\left|\dot{G}_{i i}\right|}{G_{i i}} p_{i}^{M}$ or as a time variant function $\delta_{i}(t)=\frac{o_{i}-\dot{G}_{i i} p_{i}}{G_{i i}}$ where $o_{i}>0$.

\section{REFERENCES}

[1] T. Holliday, A. Goldsmith, P. Glynn, and N. Bambos, "Distributed Power and Admission Control for Time Varying Wireless Networks: Optimality and Convergence," in Proceedings 2004 IEEE Global Telecommunications Conference - GLOBECOM'04, vol. 2, Dallas, TX, December 2004, pp. 768-774.

[2] N. Bambos, "Toward Power-Sensitive Network Architectures in Wireless Communications: Concepts, Issues, and Design Aspects," IEEE Personal Communications Magazine, vol. 5, no. 3, pp. 50-59, June 1998.

[3] V. I. Utkin, Sliding Modes in Control and Optimization. Berlin, Germany: Springer Verlag, 1992.

[4] S. Koskie and Z. Gajic, "Optimal SIR-Based Power Control in 3G Wireless CDMA Networks," in Proceedings 2003 American Control Conference - ACC'03, vol. 5, Denver, CO, December 2003, pp. 51465151.

[5] A. El-Osery and C. Abdallah, "Distributed Power Control in CDMA Cellular Systems," IEEE Antennas and Propagation Magazine, vol. 42, no. 4, pp. 152-159, August 2000.

[6] M. Lee, D. Oh, and W. Kwon, "Modified Distributed Constrained Power Control Using Reference Model in CDMA Cellular Systems," in Proceedings 42nd IEEE Conference on Decision and Control CDC'03, vol. 4, Maui, HI, December 2003, pp. 4225-4230.

[7] R. Yates, "A Framework for Uplink Power Control in Cellular Radio Systems," IEEE Journal on Selelected Areas in Communincations, vol. 13, no. 7, pp. 1341-1348, September 1995.

[8] R. A. Horn and C. A. Johnson, Matrix Analysis. Cambridge, United Kingdom: Cambridge University Press, 1985. 